\title{
Hypo-high-density Lipoprotein Cholesterolemia Caused by Evacuation after the Fukushima Daiichi Nuclear Power Plant Accident: Results from the Fukushima Health Management Survey
}

\author{
Hiroaki Satoh ${ }^{1,2}$, Tetsuya Ohira ${ }^{2,3}$, Masato Nagai ${ }^{2,3}$, Mitsuaki Hosoya ${ }^{2,4}$, Akira Sakai ${ }^{2,5}$, \\ Tsuyoshi Watanabe ${ }^{1,2}$, Akira Ohtsuru ${ }^{2,6}$, Yukihiko Kawasaki ${ }^{2,4}$, Hitoshi Suzuki ${ }^{2,7}$, \\ Atsushi Takahashi ${ }^{2,8}$, Gen Kobashi ${ }^{9}$, Kotaro Ozasa $^{10}$, Seiji Yasumura ${ }^{2,11}$, \\ Shunichi Yamashita ${ }^{2,12}$, Kenji Kamiya ${ }^{2,13}$ and Masafumi $\mathrm{Abe}^{2}$
}

\begin{abstract}
Objective The Great East Japan Earthquake and the Fukushima Daiichi nuclear disaster forced the evacuation of residents and led to many changes in the lifestyle of the evacuees. A comprehensive health check was implemented to support the prevention of lifestyle-related disease, and we analyzed changes in lipid metabolism before and after these disasters.

Methods Subjects included Japanese men and women living near the Fukushima Daiichi nuclear power plant in Fukushima Prefecture. Annual health checkups, focusing on metabolic syndromes, were conducted for persons $\geq 40$ years of age by the Heath Care Insures.

Results A total of 27,486 subjects underwent a follow-up examination after the disaster, with a mean follow-up of 1.6 years. Following the disaster, the prevalence of hypo-high-density lipoprotein (HDL) cholesterolemia increased significantly from $6.0 \%$ to $7.2 \%$. In the hypo-HDL cholesterolemia group, the body mass index (BMI), blood pressure, and LDL-C level increased significantly in men after the disaster. On the other hand, in the normal HDL-C level group, the BMI, blood pressure, glucose and lipid metabolism, and liver function were adversely affected. The decrease in HDL-C was significantly greater in evacuees than nonevacuees in the normal HDL-C level group. Furthermore, a multivariate logistic regression analysis showed that the evacuation was significantly associated with the incidence of hypo-HDL cholesterolemia.

Conclusion This is the first study to evaluate how the evacuation affected the incidence of hypo-HDL cholesterolemia and led to an increase in cardiovascular disease. This information may be important in the follow-up and lifestyle change recommendations for evacuees.
\end{abstract}

Key words: evacuee, the Great East Japan Earthquake, Fukushima Daiichi nuclear power plant accident, Fukushima Health Management Survey, hypo-high-density lipoprotein cholesterolemia, lifestyle

(Intern Med 55: 1967-1976, 2016)

(DOI: 10.2169/internalmedicine.55.6030)

\footnotetext{
${ }^{1}$ Department of Nephrology, Hypertension, Diabetology, Endocrinology, and Metabolism, Fukushima Medical University, Japan, ${ }^{2}$ Radiation Medical Science Center for the Fukushima Health Management Survey, Fukushima Medical University, Japan, ${ }^{3}$ Department of Epidemiology, Fukushima Medical University, Japan, ${ }^{4}$ Department of Pediatrics, Fukushima Medical University, Japan, ${ }^{5}$ Department of Radiation Life Sciences, Fukushima Medical University, Japan, ${ }^{6}$ Department of Radiation Health Management, Fukushima Medical University, Japan, ${ }^{7}$ Department of Cardiology and Hematology, Fukushima Medical University, Japan, ${ }^{8}$ Department of Gastroenterology and Rheumatology, Fukushima Medical University, Japan, ${ }^{9}$ Department of Planning and Management, National Institute of Radiological Sciences, Japan, ${ }^{10}$ Department of Epidemiology, Radiation Effects Research Foundation, Japan, ${ }^{11}$ Department of Public Health, Fukushima Medical University, Japan, ${ }^{12}$ Japan and Atomic Bomb Disease Institute, Nagasaki University, Japan and ${ }^{13}$ Research Institute for Radiation Biology and Medicine, Hiroshima University, Japan Received for publication June 22, 2015; Accepted for publication November 19, 2015

Correspondence to Dr. Hiroaki Satoh, hiroaki@fmu.ac.jp
} 


\section{Introduction}

The Great East Japan Earthquake occurred on March 11th, 2011. Following the earthquake, a tsunami hit the Tokyo Electric Power Company's Fukushima Daiichi nuclear power plant, resulting in a radiation hazard in Fukushima Prefecture. The Fukushima Daiichi nuclear disaster forced the evacuation of a number of towns, causing lifestyle changes and anxiety over the radiation exposure in the evacuees. Shortly after the disaster, Fukushima Prefecture launched the Fukushima Health Management Survey to investigate the effect of long-term low-dose radiation exposure caused by the disaster (1). This survey comprises a basic survey to estimate the individual radiation exposure of residents and four detailed surveys that include a comprehensive health check, thyroid ultrasonography, mental health and lifestyle survey, and survey on pregnant women and nursing mothers (1). The comprehensive health check was implemented to aid the early detection and treatment of disease, as well as the prevention of lifestyle-related diseases including diabetes, hypertension, dyslipidemia, obesity, and cardiovascular diseases.

High-density lipoprotein (HDL) promotes the efflux of cholesterol from foam cells in atherosclerotic lesions (reverse cholesterol transport). Several studies indicate that the antioxidative and anti-inflammatory properties of HDL inhibit atherogenesis $(2,3)$. A low HDL-C level is associated with an increased incidence of cardiovascular events (4) and cerebrovascular accident (CVA) (5,6). A low HDL-C level (7) is the most common lipoprotein abnormality among patients with cardiovascular disease (CVD) (8), which is now the leading cause of death worldwide (9). Large-scale protective studies have indicated that each $1 \mathrm{mg} / \mathrm{dL}$ increase in HDL-C is associated with a $2-3 \%$ decrease in the risk of CVD (10). Furthermore, a low HDL-C level commonly occurs as a result of metabolic syndrome, a cluster of factors such as insulin resistance, obesity, and hypertension, associated with an increased risk of coronary artery disease (10). These reports indicate that a low HDL-C level is one of major risk factors in cardiovascular and cerebrovascular diseases (4-6). The Japan Atherosclerosis Society (JAS) guidelines define an HDL-C level $<40 \mathrm{mg} / \mathrm{dL}$ to indicate hypoHDL cholesterolemia (11). In addition, environmental factors such as smoking, alcohol consumption and physical activity can affect the HDL-C level, and this association varies according to gender (12). The question arises as to whether this disaster influenced the HDL-C level in evacuees and non-evacuees, and if there is a significant difference in this relationship.

The primary purposes of the Fukushima Health Management Survey are to monitor the long-term health of residents, promote their future well-being, and determine whether long-term low-dose radiation exposure has health effects. Evacuees from the government-designated evacuation zone were forced to change their lifestyle, diet, exer- cise, and other personal habits. Some did not receive adequate health checkups and were anxious about their health. The comprehensive heath check attempts to review residents' health information, while assessing the incidence of various diseases and improve their health status. We herein used the information obtained from the Fukushima Health Management Survey to identify any changes in lipid metabolism, particularly the HDL-C level, before and after the disaster.

\section{Materials and Methods}

\section{Subject and study design}

The Fukushima Health Management Survey was carried out by Fukushima Medical University. Subjects included in this study were Japanese men and women living near the Fukushima Daiichi nuclear Power plant in Fukushima prefecture and residents of Tamura City, Minami Soma City, Kawamata Town, Hirono Town, Naraha Town, Tomioka Town, Kawauchi Village, Okuma Town, Futaba Town, Namie Town, Katsurao Village, Iitate Village, and Date City. The census populations of these communities were 42,085 , $71,661,16,065,5,495,7,927,15,854,3,074,11,553,7,171$, $21,551,1,582,6,584$, and 67,684 (total: 278,286) in 2010, respectively. All residents of Hirono Town, Naraha Town, Tomioka Town, Kawauchi Village, Okuma Town, Futaba Town, Namie Town, Katsurao Village, and Iitate Village, and part-time residents of Tamura City, Minami-Soma City, Kawamata Town, and Date City were forced to evacuate their homes due to a government mandate after the disaster. Since 2008, residents $\geq 40$ years of age in these communities have undergone annual health checkups by Health Care Insurers with a focus on metabolic syndromes (the target subjects of checkups was 91,554 in 2010). Between 2008 and 2010, 41,633 subjects (18,745 men and 22,888 women; mean age, 67 years) in the communities of interest participated in the health checkups.

The annual health checkups were conducted between June 2011 and March 2013 as part of the comprehensive heath check. Detailed methods of the comprehensive heath check have been previously reported (1). In brief, this comprehensive heath check performed health examinations for individuals of all ages living in the evacuation zone designated by the government who were officially registered residents at the time of the earthquake. Only residents 40-90 years of age were included in the analyses. A total of 27,486 subjects $(12,432$ men and 15,054 women; follow-up proportion: $66 \%$ ) underwent the follow-up examination following the disaster, with a mean follow-up of 1.6 years.

\section{Ethics statement}

Informed consent was obtained from the community representatives to conduct an epidemiological study according to the guidelines of the Council for International Organizations of Medical Science (13). The study was approved by 
the Fukushima Medical University Institutional Review Board (\#1916). Individuals older than 40 years of age were evaluated according to items in the Specific Health Examination, which is based on the Act on Assurance of Medical Care for Elderly People (Act No. 80, 1982). The participants in the Fukushima Health Management Survey provided their written informed consent at the follow-up survey. We obtained informed consent from all participants. To use the data before the disaster, we obtained permission from the community representatives because they have managed the data. To compare the data before and after the disaster, we used the health checkup data managed by each community. The participant consent forms were maintained in locked areas in the Fukushima Health Management Survey. The consent procedure was approved by the Fukushima Medical University Institutional Review Board.

\section{Measurements}

The examination included height, weight, abdominal circumference/body mass index (BMI), blood pressure, aspartate aminotransferase (AST), alanine aminotransferase (ALT), $\gamma$-glutamyl transpeptidase $(\gamma$-GT), triglyceride (TG), HDL-C, low-density lipoprotein cholesterol (LDL-C), hemoglobin A1c (HbA1c), fasting plasma glucose, and urine protein and sugar level measurements. The TG, HDL-C, and LDL-C levels before and after the disaster were measured using the Autoanalyzer JCA-BM8030 (JEOL Ltd.) at the laboratory of the Fukushima Preservative Service Association of Health, except for residents in Futaba Town $(n=758)$. Since the trends in the TG, HDL-C, and LDL-C levels before and after the disaster in Futaba were similar to those in other communities, we included the data of Futaba in the analyses. Additional measurements included in the examination were the serum creatinine $(\mathrm{Cr})$ level, estimated glomerular filtration rate (eGFR), uric acid (UA) level, urine testing for occult blood, and peripheral blood count, which included the red blood cell count (RBC), hematocrit (Ht), hemoglobin $(\mathrm{Hb})$, platelet count, and white blood cell count (WBC). The value for HbAlc was estimated using a National Glycohemoglobin Standardization Program equivalent value calculated using the equation $\mathrm{HbA1c}(\%)=1.019 \times$ HbA1c (JDS) $(\%)+0.30 \%$ (14).

Hypo-HDL cholesterolemia was defined as HDL-C $<40$ $\mathrm{mg} / \mathrm{dL}$ in accordance with the JAS guidelines (11). Diabetes was defined in accordance with the Japan Diabetes Society (JDS) guidelines (14): a fasting plasma glucose level $\geq 126$ $\mathrm{mg} / \mathrm{dL}(7.0 \mathrm{mmol} / \mathrm{L})$, HbA1c level $\geq 6.5 \%$, or the selfreported use of antihyperglycemic drugs.

\section{Statistical analysis}

Data are presented as the mean \pm deviation (S.D.). Changes in the data before and after the disaster were compared using the paired Student's $t$-test. An analysis of variance (ANOVA) was used to examine the differences in changes in the variables adjusted for age and sex among the evacuees and non-evacuees. These analyses were also tested after

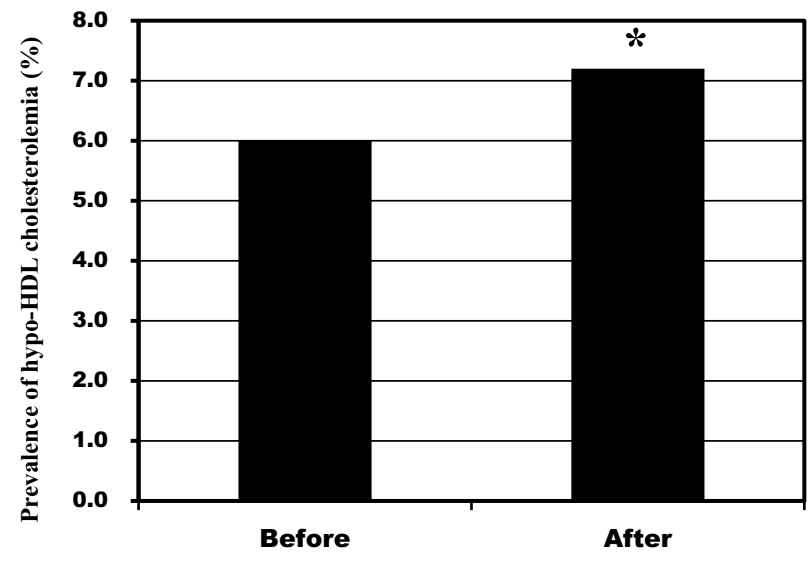

Figure. Prevalence of hypo-HDL cholesterolemia before and after the Great East Japan Earthquake. Subjects were classified into hypo-HDL cholesterolemia and no hypo-HDL cholesterolemia groups according to the Japan Atherosclerosis Society (JAS) guidelines. Hypo-HDL cholesterolemia was defined as a HDL-C level $<40 \mathrm{mg} / \mathrm{dL}$. *: $\mathbf{p}<\mathbf{0 . 0 0 0 1}$

stratifying by the HDL-C status: normal HDL-C level group and hypo-HDL cholesterolemia group at baseline. A multivariate logistic regression analysis was used to test for associations between the evacuation and other potential confounders with the incidence of a low HDL-C level. The fit of the models was evaluated using a residual analysis. The incidence of a low HDL-C level after the earthquake among evacuees and non-evacuees was compared using the MannWhitney $U$ test or $\chi^{2}$ test. The SAS version 9.3 software program (SAS Institute, Cary, USA) was used for all analyses. All probability values for statistical tests were 2-tailed and $\mathrm{p}$ values $<0.05$ were considered to be statistically significant.

\section{Results}

\section{Change in the clinical characteristics after the disas- ter}

There were 27,486 participants $(12,432$ men and 15,054 women; follow-up rate: $66 \%$; mean follow-up period: 1.6 years) who were officially registered residents at the time of the earthquake and underwent a follow-up examination after the disaster.

The HDL-C levels significantly decreased from 56.2 \pm 14.4 $\mathrm{mg} / \mathrm{dL}$ to $55.1 \pm 14.3 \mathrm{mg} / \mathrm{dL}(\mathrm{p}<0.0001)$ and $62.1 \pm 14.2 \mathrm{mg} /$ $\mathrm{dL}$ to $61.5 \pm 14 . .4 \mathrm{mg} / \mathrm{dL}(\mathrm{p}<0.0001)$ in men and women (27,486 participants; 12,432 men and 15,054 women), respectively, after the disaster. Several lines of evidence suggest that a low HDL-C level is one of major risk factors in cardiovascular and cerebrovascular diseases (4-6). In addition, the JAS guidelines (11) define an HDL-C level of $<40 \mathrm{mg} / \mathrm{dL}$ to indicate hypo-HDL cholesterolemia. Therefore, the subjects were classified into the normal HDL-C level group and hypo-HDL cholesterolemia group at base- 
Table 1. Clinical and Biochemical Characteristics of Subjects Classified by HDL-C Level before and after the Great East Japan Earthquake.

\begin{tabular}{|c|c|c|c|c|c|c|}
\hline \multicolumn{7}{|c|}{ HDL-C $<40 \mathrm{mg} / \mathrm{dL}$} \\
\hline & \multicolumn{3}{|c|}{ Men } & \multicolumn{3}{|c|}{ Women } \\
\hline & Before & After & $\mathrm{p}$ value & Before & After & $p$ value \\
\hline Number & \multicolumn{2}{|c|}{1,184} & \multicolumn{4}{|c|}{467} \\
\hline Age (years) & $67.6 \pm 10.1$ & $69.1 \pm 10.0$ & $<0.0001$ & $67.7 \pm 9.3$ & $69.2 \pm 9.3$ & $<0.0001$ \\
\hline Body weight (kg) & $66.0 \pm 9.8$ & $66.5 \pm 10.0$ & $<0.0001$ & $55.5 \pm 9.2$ & $55.6 \pm 9.4$ & 0.672 \\
\hline BMI $\left(\mathrm{kg} / \mathrm{m}^{2}\right)$ & $24.8 \pm 3.0$ & $25.0 \pm 3.1$ & $<0.0001$ & $24.8 \pm 3.5$ & $24.9 \pm 3.6$ & 0.073 \\
\hline SBP (mmHg) & $132.6 \pm 16.7$ & $134.6 \pm 15.3$ & $<0.0001$ & $132.5 \pm 16.9$ & $132.6 \pm 15.6$ & 0.969 \\
\hline $\mathrm{DBP}(\mathrm{mmHg})$ & $78.0 \pm 10.7$ & $79.2 \pm 10.1$ & 0.000 & $76.4 \pm 9.8$ & $77.2 \pm 9.9$ & 0.123 \\
\hline HbAlc $(\%)$ & $5.67 \pm 0.83$ & $5.69 \pm 0.83$ & 0.089 & $5.59 \pm 0.58$ & $5.60 \pm 0.70$ & 0.566 \\
\hline HDL-C (mg/dL) & $35.6 \pm 3.3$ & $38.2 \pm 6.3$ & $<0.0001$ & $36.1 \pm 3.0$ & $39.7 \pm 6.6$ & $<0.0001$ \\
\hline LDL-C (mg/dL) & $113.0 \pm 31.1$ & $117.4 \pm 32.0$ & $<0.0001$ & $116.2 \pm 31.2$ & $122.9 \pm 31.4$ & $<0.0001$ \\
\hline $\operatorname{AST}(\mathrm{IU} / \mathrm{L})$ & $25.9 \pm 12.5$ & $26.9 \pm 13.1$ & 0.002 & $24.8 \pm 13.1$ & $25.7 \pm 11.8$ & 0.097 \\
\hline ALT (IU/L) & $24.6 \pm 20.2$ & $26.1 \pm 17.1$ & 0.015 & $20.3 \pm 15.8$ & $21.7 \pm 15.3$ & 0.052 \\
\hline$\gamma-\mathrm{GT}(\mathrm{IU} / \mathrm{L})$ & $38.3 \pm 48.8$ & $39.4 \pm 41.5$ & 0.220 & $25.1 \pm 18.6$ & $26.5 \pm 23.5$ & 0.025 \\
\hline \multicolumn{7}{|l|}{ Smoking habit (\%) } \\
\hline Yes & 32.4 & 26.9 & \multirow{2}{*}{$<0.0001$} & 5.0 & 3.7 & \multirow{2}{*}{0.070} \\
\hline No & 67.6 & 73.1 & & 95.0 & 96.3 & \\
\hline \multicolumn{7}{|l|}{ Drinking } \\
\hline Everyday & 24.4 & 23.1 & \multirow{4}{*}{0.122} & 1.7 & 0.7 & \multirow{4}{*}{0.495} \\
\hline Sometimes & 25.1 & 24.0 & & 10.0 & 11.2 & \\
\hline Little & 22.5 & 25.4 & & 30.0 & 29.0 & \\
\hline Never & 28.1 & 27.5 & & 38.3 & 59.1 & \\
\hline \multicolumn{7}{|c|}{ HDL-C $\geq 40 \mathrm{mg} / \mathrm{dL}$} \\
\hline & \multicolumn{3}{|c|}{ Men } & \multicolumn{3}{|c|}{ Women } \\
\hline & Before & After & $\mathrm{p}$ value & Before & After & $\mathrm{p}$ value \\
\hline Number & \multicolumn{2}{|c|}{11,248} & \multicolumn{4}{|c|}{14,587} \\
\hline Age (years) & $66.8 \pm 9.4$ & $68.3 \pm 9.4$ & $<0.0001$ & $65.7 \pm 9.4$ & $67.2 \pm 9.4$ & $<0.0001$ \\
\hline Body weight (kg) & $62.1 \pm 9.6$ & $63.0 \pm 9.8$ & $<0.0001$ & $52.6 \pm 8.4$ & $53.0 \pm 8.7$ & $<0.0001$ \\
\hline BMI $\left(\mathrm{kg} / \mathrm{m}^{2}\right)$ & $23.5 \pm 3.0$ & $23.9 \pm 3.1$ & $<0.0001$ & $23.3 \pm 3.4$ & $23.6 \pm 3.5$ & $<0.0001$ \\
\hline $\mathrm{SBP}(\mathrm{mmHg})$ & $132.9 \pm 16.6$ & $134.6 \pm 15.8$ & $<0.0001$ & $130.5 \pm 17.1$ & $132.2 \pm 16.3$ & $<0.0001$ \\
\hline DBP (mmHg) & $78.4 \pm 10.2$ & $79.5 \pm 10.1$ & $<0.0001$ & $75.9 \pm 10.2$ & $77.2 \pm 9.9$ & $<0.0001$ \\
\hline $\mathrm{HbAlc}(\%)$ & $5.52 \pm 0.67$ & $5.55 \pm 0.74$ & $<0.0001$ & $5.50 \pm 0.59$ & $5.49 \pm 0.60$ & $<0.0001$ \\
\hline HDL-C (mg/dL) & $58.3 \pm 13.4$ & $56.9 \pm 13.7$ & $<0.0001$ & $62.9 \pm 13.7$ & $62.2 \pm 14.0$ & $<0.0001$ \\
\hline LDL-C (mg/dL) & $116.0 \pm 29.4$ & $118.0 \pm 30.3$ & $<0.0001$ & $124.9 \pm 29.4$ & $126.3 \pm 30.7$ & $<0.0001$ \\
\hline $\operatorname{AST}(\mathrm{IU} / \mathrm{L})$ & $26.6 \pm 11.9$ & $27.2 \pm 12.5$ & $<0.0001$ & $23.8 \pm 8.9$ & $24.1 \pm 9.8$ & $<0.0001$ \\
\hline ALT (IU/L) & $22.8 \pm 14.3$ & $24.4 \pm 16.5$ & $<0.0001$ & $18.7 \pm 11.2$ & $19.6 \pm 13.1$ & $<0.0001$ \\
\hline$\gamma-\mathrm{GT}(\mathrm{IU} / \mathrm{L})$ & $45.3 \pm 61.0$ & $47.6 \pm 64.4$ & $<0.0001$ & $23.6 \pm 27.1$ & $24.6 \pm 24.1$ & $<0.0001$ \\
\hline \multicolumn{7}{|l|}{ Smoking habit (\%) } \\
\hline Yes & 23.4 & 20.3 & \multirow{2}{*}{$<0.0001$} & 3.7 & 3.4 & \multirow{2}{*}{$<0.0001$} \\
\hline No & 76.7 & 79.7 & & 96.3 & 96.6 & \\
\hline \multicolumn{7}{|l|}{ Drinking } \\
\hline Everyday & 48.7 & 47.7 & \multirow{4}{*}{$<0.0001$} & 4.9 & 4.9 & \multirow{4}{*}{$<0.0001$} \\
\hline Sometimes & 22.4 & 21.6 & & 17.1 & 15.7 & \\
\hline Little & 13.8 & 14.9 & & 29.3 & 29.8 & \\
\hline Never & 15.0 & 15.8 & & 48.7 & 49.6 & \\
\hline
\end{tabular}

Data are presented as mean \pm S.D

BMI: body mass index, SBP: systolic blood pressure, DBP: diastolic blood pressure, HbA1c: hemoglobin A1c, HDL-C: high density lipoprotein cholesterol, LDL-C: low density lipoprotein cholesterol, AST: aspartate aminotransferase, ALT: alanine aminotransferase, $\gamma$-GT: $\gamma$-glutamyl transpeptidase

line. The prevalence of hypo-HDL cholesterolemia before and after the Great East Japan Earthquake is shown in Figure. Following the disaster, the prevalence of hypo-HDL cholesterolemia significantly increased from $6.0 \%$ to $7.2 \%$ $(\mathrm{p}<0.0001)$.

In the hypo-HDL cholesterolemia group (1,651 participants; 1,184 men and 467 women), the HDL-C level significantly increased from $35.6 \pm 3.3 \mathrm{mg} / \mathrm{dL}$ to $38.2 \pm 6.3 \mathrm{mg} / \mathrm{dL}(\mathrm{p}$ $<0.0001)$ and $36.1 \pm 3.0 \mathrm{mg} / \mathrm{dL}$ to $39.7 \pm 6.6 \mathrm{mg} / \mathrm{dL} \quad(\mathrm{p}<$ 0.0001 ) in men and women, respectively, after the disaster. In addition, the ratio of smoking habit significantly decreased from $32.4 \%$ to $26.9 \% \quad(\mathrm{p}<0.0001)$ in only men. However, the BMI, blood pressure, AST, and ALT also significantly increased in both men and women after the disas- ter (Table 1). In the normal HDL-C level group (25,835 participants; 11,248 men and 14,587 women), the HDL-C level significantly decreased after the disaster from $58.3 \pm 13.4 \mathrm{mg} /$ $\mathrm{dL}$ to $56.9 \pm 13.7 \mathrm{mg} / \mathrm{dL}(\mathrm{p}<0.0001)$ and $62.9 \pm 13.7 \mathrm{mg} / \mathrm{dL}$ to $62.2 \pm 14.0 \mathrm{mg} / \mathrm{dL}(\mathrm{p}<0.0001)$ in men and women, respectively, whereas the ratio of smoking habit significantly decreased from $23.4 \%$ to $20.3 \%(\mathrm{p}<0.0001)$ and $3.7 \%$ to $3.4 \%$ $(\mathrm{p}<0.0001)$ in men and women, respectively. In addition, the BMI, blood pressure, LDL-C, AST, ALT, and $\gamma$-GT levels also increased significantly in men and women $(\mathrm{p}<0.0001$ for both) after the disaster. 
Table 2. Clinical and Biochemical Characteristics among Non-evacuees and Evacuees before and after the Great East Japan Eathquake.

\begin{tabular}{|c|c|c|c|c|c|c|}
\hline \multicolumn{7}{|c|}{$\mathrm{HDL}-\mathrm{C}<40 \mathrm{mg} / \mathrm{dL}$} \\
\hline \multicolumn{7}{|c|}{ Men } \\
\hline & \multicolumn{3}{|c|}{ Evacuees } & \multicolumn{3}{|c|}{ Non-evacuees } \\
\hline & Before & After & $\mathrm{p}$ value & Before & After & $\mathrm{p}$ value \\
\hline Number & \multicolumn{2}{|c|}{404} & \multicolumn{4}{|c|}{780} \\
\hline Age (years) & $66.7 \pm 10.3$ & $68.3 \pm 10.3$ & $<0.0001$ & $68.0 \pm 9.9$ & $69.5 \pm 9.9$ & $<.0001$ \\
\hline Body weight (kg) & $66.7 \pm 9.5$ & $68.1 \pm 10.0$ & $<0.0001$ & $65.6 \pm 9.9$ & $65.6 \pm 9.9$ & 0.527 \\
\hline BMI $\left(\mathrm{kg} / \mathrm{m}^{2}\right)$ & $25.0 \pm 2.8$ & $25.6 \pm 3.0$ & $<0.0001$ & $24.7 \pm 3.0$ & $24.8 \pm 3.0$ & 0.186 \\
\hline SBP (mmHg) & $132.4 \pm 17.4$ & $135.5 \pm 15.4$ & 0.000 & $132.7 \pm 16.3$ & $134.1 \pm 15.2$ & 0.012 \\
\hline DBP (mmHg) & $77.4 \pm 10.4$ & $80.3 \pm 9.5$ & $<0.0001$ & $78.3 \pm 10.8$ & $78.7 \pm 10.4$ & 0.333 \\
\hline $\mathrm{HbAlc}(\%)$ & $5.73 \pm 0.96$ & $5.74 \pm 0.80$ & 0.767 & $5.63 \pm 0.75$ & $5.67 \pm 0.85$ & 0.058 \\
\hline HDL-C (mg/dL) & $35.6 \pm 3.5$ & $37.8 \pm 6.7$ & $<0.0001$ & $35.6 \pm 3.2$ & $38.4 \pm 6.1$ & $<.0001$ \\
\hline LDL-C (mg/dL) & $111.4 \pm 33.2$ & $117.7 \pm 34.9$ & $<.0001$ & $113.7 \pm 30.0$ & $117.2 \pm 30.4$ & $<.0001$ \\
\hline AST (IU/L) & $27.5 \pm 16.8$ & $28.9 \pm 16.2$ & 0.024 & $25.1 \pm 9.4$ & $25.9 \pm 10.9$ & 0.038 \\
\hline ALT (IU/L) & $26.7 \pm 17.3$ & $29.8 \pm 20.6$ & 0.000 & $23.6 \pm 21.4$ & $24.1 \pm 14.6$ & 0.462 \\
\hline$\gamma-\mathrm{GT}(\mathrm{IU} / \mathrm{L})$ & $42.6 \pm 60.1$ & $44.9 \pm 52.5$ & 0.267 & $36.1 \pm 41.6$ & $36.6 \pm 34.3$ & 0.569 \\
\hline \multicolumn{7}{|l|}{ Smoking habit (\%) } \\
\hline Yes & 32.0 & 27.5 & $<0.0001$ & 32.6 & 26.6 & $<0.0001$ \\
\hline No & 68.1 & 72.5 & & 67.4 & 73.4 & \\
\hline \multicolumn{7}{|l|}{ Drinking } \\
\hline Everyday & 23.1 & 21.4 & 0.557 & 25.0 & 24.0 & 0.354 \\
\hline Sometimes & 24.4 & 23.2 & & 25.4 & 24.4 & \\
\hline Little & 20.5 & 23.4 & & 23.5 & 26.4 & \\
\hline Never & 32.0 & 32.0 & & 26.2 & 25.3 & \\
\hline \multicolumn{7}{|c|}{ Women } \\
\hline & \multicolumn{3}{|c|}{ Evacuees } & \multicolumn{3}{|c|}{ Non-evacuees } \\
\hline & Before & After & $\mathrm{p}$ value & Before & After & $\mathrm{p}$ value \\
\hline Number & \multicolumn{2}{|c|}{160} & \multicolumn{4}{|c|}{307} \\
\hline Age (years) & $67.1 \pm 9.8$ & $68.8 \pm 9.8$ & $<.0001$ & $68.0 \pm 9.0$ & $69.4 \pm 9.0$ & $<.0001$ \\
\hline Body weight (kg) & $55.8 \pm 10.1$ & $56.0 \pm 10.1$ & 0.406 & $55.4 \pm 8.8$ & $55.4 \pm 9.0$ & 0.834 \\
\hline $\operatorname{BMI}\left(\mathrm{kg} / \mathrm{m}^{2}\right)$ & $24.9 \pm 3.7$ & $25.1 \pm 3.7$ & 0.043 & $24.8 \pm 3.5$ & $24.8 \pm 3.6$ & 0.548 \\
\hline SBP (mmHg) & $132.4 \pm 17.7$ & $132.0 \pm 15.7$ & 0.616 & $132.6 \pm 16.5$ & $133.0 \pm 15.5$ & 0.657 \\
\hline DBP (mmHg) & $75.6 \pm 8.7$ & $77.5 \pm 9.4$ & 0.035 & $76.8 \pm 10.3$ & $77.0 \pm 10.2$ & 0.680 \\
\hline $\mathrm{HbA} 1 \mathrm{c}(\%)$ & $5.69 \pm 0.72$ & $5.74 \pm 0.95$ & 0.121 & $5.54 \pm 0.50$ & $5.52 \pm 0.52$ & 0.237 \\
\hline HDL-C (mg/dL) & $36.3 \pm 2.7$ & $40.1 \pm 7.5$ & $<.0001$ & $36.1 \pm 3.1$ & $39.5 \pm 6.2$ & $<.0001$ \\
\hline LDL-C (mg/dL) & $117.5 \pm 31.2$ & $124.9 \pm 31.3$ & 0.002 & $115.5 \pm 31.2$ & $121.9 \pm 31.4$ & 0.000 \\
\hline AST (IU/L) & $24.3 \pm 9.3$ & $26.4 \pm 13.9$ & 0.022 & $25.0 \pm 14.6$ & $25.3 \pm 10.6$ & 0.663 \\
\hline ALT (IU/L) & $20.7 \pm 12.8$ & $24.1 \pm 18.9$ & 0.006 & $20.1 \pm 17.1$ & $20.4 \pm 12.8$ & 0.666 \\
\hline$\gamma-\mathrm{GT}(\mathrm{IU} / \mathrm{L})$ & $25.0 \pm 18.6$ & $28.9 \pm 26.0$ & 0.003 & $25.1 \pm 18.6$ & $25.2 \pm 22.0$ & 0.800 \\
\hline \multicolumn{7}{|l|}{ Smoking habit (\%) } \\
\hline Yes & 6.5 & 5.1 & \multirow{2}{*}{0.500} & 4.2 & 2.9 & \multirow{2}{*}{0.219} \\
\hline No & 93.5 & 94.9 & & 95.8 & 97.1 & \\
\hline \multicolumn{7}{|l|}{ Drinking } \\
\hline Everyday & 2.6 & 0.6 & \multirow{4}{*}{0.364} & 1.3 & 0.7 & \multirow{4}{*}{0.905} \\
\hline Sometimes & 13.7 & 13.9 & & 8.1 & 9.8 & \\
\hline Little & 21.6 & 20.9 & & 34.2 & 33.2 & \\
\hline Never & 62.1 & 64.6 & & 56.4 & 56.4 & \\
\hline
\end{tabular}

\section{Change in the clinical characteristics among non- evacuees and evacuees after the disaster}

Next, the effects of the evacuation on lipid metabolism were examined. Among the men (12,432 participants; 4,275 evacuees and 8,157 non-evacuees), the HDL-C levels significantly decreased from $56.7 \pm 14.6 \mathrm{mg} / \mathrm{dL}$ to $54.2 \pm 14.0 \mathrm{mg} / \mathrm{dL}$ $(\mathrm{p}<0.0001)$ and $55.9 \pm 14.3 \mathrm{mg} / \mathrm{dL}$ to $55.6 \pm 14.4 \mathrm{mg} / \mathrm{dL}(\mathrm{p}=$ 0.0060 ) in evacuees and non-evacuees, respectively, after the disaster. Therefore, the subjects were classified into the normal HDL-C level group and hypo-HDL cholesterolemia group at baseline. Among men in the hypo-HDLcholesterolemia group (404 evacuees and 780 non-evacuees), the HDL-C levels significantly increased from $35.6 \pm 3.5 \mathrm{mg} /$ $\mathrm{dL}$ to $37.8 \pm 6.7 \mathrm{mg} / \mathrm{dL}(\mathrm{p}<0.0001)$ and $35.6 \pm 3.2 \mathrm{mg} / \mathrm{dL}$ to $38.4 \pm 6.1 \mathrm{mg} / \mathrm{dL}(\mathrm{p}<0.0001)$ in evacuees and non-evacuees, respectively. In addition, the ratio of smoking habit significantly decreased from $32.0 \%$ to $27.5 \% \quad(\mathrm{p}<0.0001)$ and $32.6 \%$ to $26.6 \%(\mathrm{p}<0.0001)$ in evacuees and non-evacuees, respectively (Table 2). Although the change in the HDL-C levels did not differ significantly among evacuees and nonevacuees, increases in the BMI $(\mathrm{p}<0.0001)$, diastolic blood pressure $(\mathrm{p}=0.019)$, and AST level $(\mathrm{p}=0.029)$ were significantly greater in evacuees than non-evacuees (Table 3). Among the women (15,054 participants; 5,396 evacuees and 9,658 non-evacuees), the HDL-C levels significantly decreased from $62.3 \pm 14.2 \mathrm{mg} / \mathrm{dL}$ to $60.8 \pm 14.3 \mathrm{mg} / \mathrm{dL}(\mathrm{p}<$ 0.0001 ) in evacuees after the disaster, however, the HDL-C levels did not significantly change $(61.9 \pm 14.2 \mathrm{mg} / \mathrm{dL}$ to 61.9 $\pm 14.9 \mathrm{mg} / \mathrm{dL} ; \mathrm{p}=0.7282$ ) in non-evacuees. The subjects were then classified into the normal HDL-C group and hypo-HDL cholesterolemia group at baseline. Among women in the 
Table 2. continued

\begin{tabular}{|c|c|c|c|c|c|c|}
\hline \multicolumn{7}{|c|}{ HDL-C $\geq 40 \mathrm{mg} / \mathrm{dL}$} \\
\hline \multicolumn{7}{|c|}{ Men } \\
\hline & \multicolumn{3}{|c|}{ Evacuees } & \multicolumn{3}{|c|}{ Non-evacuees } \\
\hline & Before & After & $\mathrm{p}$ value & Before & After & $p$ value \\
\hline Number & \multicolumn{2}{|c|}{3,871} & \multicolumn{4}{|c|}{7,377} \\
\hline Age (years) & $66.6 \pm 9.5$ & $68.2 \pm 9.5$ & $<0.0001$ & $66.9 \pm 9.3$ & $68.3 \pm 9.3$ & $<0.0001$ \\
\hline Body weight $(\mathrm{kg})$ & $62.5 \pm 9.7$ & $64.3 \pm 9.9$ & $<0.0001$ & $61.8 \pm 9.4$ & $62.3 \pm 9.6$ & $<0.0001$ \\
\hline $\operatorname{BMI}\left(\mathrm{kg} / \mathrm{m}^{2}\right)$ & $23.6 \pm 3.1$ & $24.4 \pm 3.1$ & $<0.0001$ & $23.4 \pm 3.0$ & $23.6 \pm 3.0$ & $<0.0001$ \\
\hline SBP (mmHg) & $133.7 \pm 17.4$ & $134.8 \pm 15.4$ & $<0.0001$ & $132.5 \pm 16.2$ & $134.5 \pm 16.0$ & $<0.0001$ \\
\hline DBP (mmHg) & $78.2 \pm 10.3$ & $79.9 \pm 9.9$ & $<0.0001$ & $78.5 \pm 10.1$ & $79.2 \pm 10.2$ & $<0.0001$ \\
\hline HbAlc (\%) & $5.55 \pm 0.69$ & $5.59 \pm 0.79$ & $<0.0001$ & $5.51 \pm 0.66$ & $5.52 \pm 0.71$ & $<0.0001$ \\
\hline HDL-C (mg/dL) & $58.9 \pm 13.5$ & $55.9 \pm 13.4$ & $<0.0001$ & $58.0 \pm 13.3$ & $57.5 \pm 13.8$ & $<0.0001$ \\
\hline LDL-C (mg/dL) & $115.6 \pm 29.9$ & $120.5 \pm 31.1$ & $<0.0001$ & $116.2 \pm 29.1$ & $116.7 \pm 29.8$ & 0.07 \\
\hline $\operatorname{AST}(\mathrm{IU} / \mathrm{L})$ & $27.2 \pm 14.1$ & $27.9 \pm 12.0$ & 0.003 & $26.3 \pm 10.5$ & $26.9 \pm 12.7$ & $<0.0001$ \\
\hline ALT (IU/L) & $23.6 \pm 16.1$ & $26.5 \pm 18.3$ & $<0.0001$ & $22.4 \pm 13.2$ & $23.3 \pm 15.3$ & $<0.0001$ \\
\hline$\gamma$-GT (IU/L) & $46.9 \pm 64.1$ & $49.8 \pm 57.0$ & 0.0003 & $44.5 \pm 59.3$ & $46.5 \pm 67.9$ & 0.0012 \\
\hline \multicolumn{7}{|l|}{ Smoking habit (\%) } \\
\hline Yes & 24.6 & 21.4 & \multirow{2}{*}{$<0.0001$} & 22.7 & 19.8 & \multirow{2}{*}{$<0.0001$} \\
\hline No & 75.4 & 78.6 & & 77.3 & 80.2 & \\
\hline \multicolumn{7}{|l|}{ Drinking } \\
\hline Everyday & 48.8 & 46.9 & \multirow{4}{*}{$<0.0001$} & 48.7 & 48.1 & \multirow{4}{*}{$<0.0001$} \\
\hline Sometimes & 22.7 & 21.1 & & 22.3 & 21.8 & \\
\hline Little & 13.4 & 14.7 & & 14.0 & 14.9 & \\
\hline Never & 15.1 & 17.3 & & 15.0 & 15.1 & \\
\hline \multicolumn{7}{|c|}{ Women } \\
\hline & \multicolumn{3}{|c|}{ Evacuees } & \multicolumn{3}{|c|}{ Non-evacuees } \\
\hline & Before & After & $\mathrm{p}$ value & Before & After & $\mathrm{p}$ value \\
\hline Number & \multicolumn{2}{|c|}{5,236} & \multicolumn{4}{|c|}{9,351} \\
\hline Age (years) & $65.9 \pm 9.9$ & $67.5 \pm 9.9$ & $<0.0001$ & $65.6 \pm 9.1$ & $67.1 \pm 9.1$ & $<0.0001$ \\
\hline Body weight $(\mathrm{kg})$ & $53.0 \pm 8.6$ & $53.8 \pm 8.8$ & $<0.0001$ & $52.3 \pm 8.4$ & $52.5 \pm 8.6$ & $<0.0001$ \\
\hline BMI $\left(\mathrm{kg} / \mathrm{m}^{2}\right)$ & $23.5 \pm 3.5$ & $24.0 \pm 3.6$ & $<0.0001$ & $23.2 \pm 3.4$ & $23.4 \pm 3.5$ & $<0.0001$ \\
\hline SBP (mmHg) & $131.1 \pm 17.7$ & $131.8 \pm 16.1$ & 0.005 & $130.2 \pm 16.8$ & $132.5 \pm 16.4$ & $<0.0001$ \\
\hline DBP (mmHg) & $75.4 \pm 10.3$ & $77.2 \pm 9.9$ & $<0.0001$ & $76.1 \pm 10.1$ & $77.2 \pm 9.9$ & $<0.0001$ \\
\hline $\mathrm{HbAlc}(\%)$ & $5.54 \pm 0.64$ & $5.51 \pm 0.66$ & $<0.0001$ & $5.48 \pm 0.55$ & $5.47 \pm 0.57$ & 0.004 \\
\hline HDL-C (mg/dL) & $63.1 \pm 13.7$ & $61.4 \pm 14.0$ & $<0.0001$ & $62.8 \pm 13.7$ & $62.7 \pm 13.9$ & $<0.0001$ \\
\hline LDL-C (mg/dL) & $124.1 \pm 29.7$ & $127.9 \pm 32.1$ & $<0.0001$ & $125.3 \pm 29.2$ & $125.5 \pm 29.9$ & 0.592 \\
\hline AST (IU/L) & $23.9 \pm 10.0$ & $24.5 \pm 10.6$ & $<0.0001$ & $23.7 \pm 8.2$ & $23.9 \pm 9.3$ & 0.006 \\
\hline ALT (IU/L) & $19.0 \pm 12.5$ & $20.5 \pm 14.9$ & $<0.0001$ & $18.5 \pm 10.4$ & $19.1 \pm 11.9$ & $<0.0001$ \\
\hline$\gamma-\mathrm{GT}(\mathrm{IU} / \mathrm{L})$ & $23.8 \pm 31.3$ & $25.6 \pm 26.5$ & $<0.0001$ & $23.5 \pm 24.4$ & $24.0 \pm 22.7$ & 0.009 \\
\hline \multicolumn{7}{|l|}{ Smoking habit (\%) } \\
\hline Yes & 4.4 & 4.2 & \multirow{2}{*}{0.248} & 3.4 & 2.9 & \multirow{2}{*}{$<0.0001$} \\
\hline No & 95.6 & 95.8 & & 96.6 & 97.1 & \\
\hline \multicolumn{7}{|l|}{ Drinking } \\
\hline Everyday & 5.7 & 5.9 & \multirow{4}{*}{$<0.0001$} & 4.5 & 4.3 & \\
\hline Sometimes & 18.2 & 15.3 & & 16.5 & 15.9 & \\
\hline Little & 27.2 & 26.6 & & 30.4 & 31.5 & 0.024 \\
\hline Never & 48.9 & 52.3 & & 48.7 & 48.2 & \\
\hline
\end{tabular}

Data are presented as mean \pm S.D.

BMI: body mass index, SBP: systolic blood pressure, DBP: diastolic blood pressure, HbAlc: hemoglobin Alc, HDL-C: high density lipoprotein cholesterol, LDL-C: low density lipoprotein cholesterol, AST: aspartate aminotransferase, ALT: alanine aminotransferase, $\gamma$-GT: $\gamma$-glutamyl transpeptidase

hypo-HDL cholesterolemia group (160 evacuees and 307 non-evacuees), the HDL-C levels significantly increased from $36.3 \pm 2.7 \mathrm{mg} / \mathrm{dL}$ to $40.1 \pm 7.5 \mathrm{mg} / \mathrm{dL}(\mathrm{p}<0.0001)$ and $36.1 \pm 3.1 \mathrm{mg} / \mathrm{dL}$ to $39.5 \pm 6.2 \mathrm{mg} / \mathrm{dL}(\mathrm{p}<0.0001)$ in evacuees and non-evacuees, respectively, whereas the ratio of smoking habit did not significantly change in evacuees and nonevacuees. Additionally, the changes in the HDL-C levels and other risk factors did not differ significantly among evacuees and non-evacuees. On the other hand, among men in the normal HDL-C level group (3,871 evacuees and 7,377 nonevacuees), the HDL-C levels significantly decreased from $58.9 \pm 13.5 \mathrm{mg} / \mathrm{dL}$ to $55.9 \pm 13.4 \mathrm{mg} / \mathrm{dL}(\mathrm{p}<0.0001)$ and $58.0 \pm$ $13.3 \mathrm{mg} / \mathrm{dL}$ to $57.5 \pm 13.8 \mathrm{mg} / \mathrm{dL}(\mathrm{p}<0.0001)$ in evacuees and non-evacuees, respectively, whereas the ratio of smoking habit significantly decreased from $24.6 \%$ to $21.4 \%$ ( $\mathrm{p}<$
$0.0001)$ and $22.7 \%$ to $19.8 \%(\mathrm{p}<0.0001)$ in evacuees and non-evacuees, respectively (Table 2). Moreover, the decrease in the HDL-C levels was greater $(p<0.0001)$ in evacuees than non-evacuees (Table 3), and increases in the BMI $(\mathrm{p}<$ $0.0001)$, diastolic blood pressure $(\mathrm{p}=0.032)$, HbA1c $(\mathrm{p}=$ 0.016), LDL-C $(\mathrm{p}<0.0001)$, and ALT levels $(\mathrm{p}<0.0001)$ were significantly greater in evacuees than non-evacuees. Among women in the normal HDL-C level group (5,236 evacuees and 9,351 non-evacuees), the HDL-C levels significantly increased from $63.1 \pm 13.7 \mathrm{mg} / \mathrm{dL}$ to $61.4 \pm 14.0 \mathrm{mg} / \mathrm{dL} \quad(\mathrm{p}<$ $0.0001)$ and $62.8 \pm 13.7 \mathrm{mg} / \mathrm{dL}$ to $62.7 \pm 13.9 \mathrm{mg} / \mathrm{dL}(\mathrm{p}<$ 0.0001 ) in evacuees and non-evacuees, respectively. In addition, the ratio of smoking habit significantly decreased from $3.4 \%$ to $2.9 \%(\mathrm{p}<0.0001)$ in only non-evacuees, respectively (Table 2). Additionally, a significantly greater decrease in 
Table 3. Changes in Clinical and Biochemical Characteristics among Non-evacuees and Evacuees before and after the Great East Japan Earthquake.

\begin{tabular}{|c|c|c|c|c|c|c|}
\hline & \multicolumn{6}{|c|}{ HDL-C $<40 \mathrm{mg} / \mathrm{dL}$} \\
\hline & \multicolumn{3}{|c|}{ Men } & \multicolumn{3}{|c|}{ Women } \\
\hline & Evacuees & Non-evacuees & $\mathrm{p}$ value & Evacuees & Non-evacuees & $\mathrm{p}$ value \\
\hline Number & 404 & 780 & & 160 & 307 & \\
\hline Age (years) & 1.6 & 1.4 & 0.002 & 1.7 & 1.4 & 0.000 \\
\hline Body weight (kg) & 1.5 & 0.1 & $<0.0001$ & 0.2 & -0.03 & 0.406 \\
\hline BMI $\left(\mathrm{kg} / \mathrm{m}^{2}\right)$ & 0.6 & 0.1 & $<0.0001$ & 0.2 & 0.0 & 0.148 \\
\hline SBP (mmHg) & 3.1 & 1.4 & 0.078 & -0.7 & 0.4 & 0.495 \\
\hline DBP $(\mathrm{mmHg})$ & 2.9 & 0.4 & $<0.0001$ & 1.8 & 0.3 & 0.154 \\
\hline $\mathrm{HbAlc}(\%)$ & 0.01 & 0.04 & 0.391 & 0.06 & -0.02 & 0.063 \\
\hline HDL-C (mg/dL) & 2.1 & 2.8 & 0.101 & 3.4 & 3.3 & 0.893 \\
\hline LDL-C (mg/dL) & 6.5 & 3.6 & 0.081 & 7.7 & 6.4 & 0.648 \\
\hline $\mathrm{AST}(\mathrm{IU} / \mathrm{L})$ & 1.4 & 0.8 & 0.374 & 2.2 & 0.3 & 0.118 \\
\hline ALT (IU/L) & 3.2 & 0.6 & 0.029 & 3.5 & 0.4 & 0.050 \\
\hline \multirow[t]{4}{*}{$\gamma-\mathrm{GT}(\mathrm{IU} / \mathrm{L})$} & 2.4 & 0.5 & 0.416 & 4.2 & 0.2 & 0.012 \\
\hline & \multicolumn{6}{|c|}{$\mathrm{HDL}-\mathrm{C} \geq 40 \mathrm{mg} / \mathrm{dL}$} \\
\hline & \multicolumn{3}{|c|}{ Men } & \multicolumn{3}{|c|}{ Women } \\
\hline & Evacuees & Non-evacuees & $p$ value & Evacuees & Non-evacuees & $\mathrm{p}$ value \\
\hline Number & 3,871 & 7,377 & & 5,236 & 9,351 & \\
\hline Age (years) & 1.6 & 1.4 & $<.0001$ & 1.6 & 1.5 & $<0.0001$ \\
\hline Body weight (kg) & 1.8 & 0.5 & $<.0001$ & 0.8 & 0.2 & $<0.0001$ \\
\hline BMI $\left(\mathrm{kg} / \mathrm{m}^{2}\right)$ & 0.8 & 0.2 & $<.0001$ & 0.5 & 0.2 & $<0.0001$ \\
\hline SBP (mmHg) & 1.1 & 1.9 & 0.013 & 0.7 & 2.2 & $<0.0001$ \\
\hline DBP $(\mathrm{mmHg})$ & 1.7 & 0.8 & $<.0001$ & 1.8 & 1.0 & $<.0001$ \\
\hline $\mathrm{HbAlc}(\%)$ & 0.04 & 0.02 & 0.016 & -0.01 & -0.03 & 0.019 \\
\hline HDL-C (mg/dL) & -3.0 & -0.6 & $<.0001$ & -0.1 & -1.7 & $<0.0001$ \\
\hline LDL-C (mg/dL) & 4.9 & 0.5 & $<.0001$ & 0.1 & 3.4 & $<0.0001$ \\
\hline $\operatorname{AST}(\mathrm{IU} / \mathrm{L})$ & 0.7 & 0.6 & 0.737 & 0.2 & 0.6 & 0.016 \\
\hline ALT (IU/L) & 2.9 & 1.0 & $<.0001$ & 0.6 & 1.6 & $<0.0001$ \\
\hline$\gamma$-GT (IU/L) & 2.9 & 2.0 & 0.332 & 1.9 & 0.4 & $<0.0001$ \\
\hline
\end{tabular}

Data are presented as mean \pm S.D.

$\mathrm{P}$ value comparing the changes in the evacuee group to the changes in the non-evacuee group between before and after the earthquake.

BMI: body mass index, SBP: systolic blood pressure, DBP: diastolic blood pressure, HbA1c: hemoglobin A1c, HDL-C: high density lipoprotein cholesterol, LDL-C: low density lipoprotein cholesterol, AST: aspartate aminotransferase, ALT: alanine aminotransferase, $\gamma$-GT: $\gamma$-glutamyl transpeptidase

Table 4. Multivariate Logistic Regression Analysis of Factors Influencing the Incidence of Hypo-HDL Cholesterolemia after the Great East Japan Earthquake.

\begin{tabular}{|c|c|c|c|c|c|c|}
\hline \multirow[b]{2}{*}{ Model } & \multicolumn{2}{|l|}{ Total } & \multicolumn{2}{|l|}{ Men } & \multicolumn{2}{|l|}{ Women } \\
\hline & OR $(95 \% \mathrm{CI})$ & $\mathrm{p}$ value & OR $(95 \% \mathrm{CI})$ & $\mathrm{p}$ value & OR $(95 \% \mathrm{CI})$ & $\mathrm{p}$ value \\
\hline Evacuation & $1.387(1.214-1.584)$ & $<0.0001$ & $1.413(1.198-1.667)$ & $<0.0001$ & $1.351(1.078-1.693)$ & 0.0089 \\
\hline Age (years) & $1.019(1.011-1.026)$ & $<0.0001$ & $1.017(1.008-1.027)$ & 0.0004 & $1.021(1.008-1.035)$ & 0.0018 \\
\hline $\operatorname{BMI}\left(\mathrm{kg} / \mathrm{m}^{2}\right)$ & $1.106(1.084-1.129)$ & $<0.0001$ & $1.129(1.099-1.159)$ & $<0.0001$ & $1.074(1.040-1.109)$ & $<0.0001$ \\
\hline Change in BMI $\left(1 \mathrm{~kg} / \mathrm{m}^{2}\right)$ & $1.205(1.141-1.272)$ & $<0.0001$ & $1.197(1.116-1.285)$ & $<0.0001$ & $1.222(1.121-1.332)$ & $<0.0001$ \\
\hline $\mathrm{SBP}(\mathrm{mmHg})$ & $1.001(0.997-1.005)$ & 0.5995 & $1.002(0.997-1.007)$ & 0.5242 & $1.000(0.993-1.007)$ & 0.9624 \\
\hline ALT (IU/L) & $1.384(1.166-1.643)$ & 0.0002 & $1.238(1.004-1.527)$ & 0.0456 & $1.657(1.224-2.243)$ & 0.0011 \\
\hline$\gamma$-GT (IU/L) & $0.791(0.695-0.900)$ & 0.0004 & $0.766(0.660-0.890)$ & 0.0005 & $0.871(0.672-1.129)$ & 0.2971 \\
\hline Diabetes & $1.284(1.048-1.573)$ & 0.0157 & $1.237(0.973-1.573)$ & 0.0827 & $1.365(0.932-1.999)$ & 0.1097 \\
\hline
\end{tabular}

Data are presented as odds ratio (95\% confidence interval).

OR: odds ratio, CI: confidence interval, BMI: body mass index, SBP: systolic blood pressure, ALT: alanine aminotransferase, $\gamma$-GT: $\gamma$-glutamyl transpeptidase

Diabetes was defined as a fasting plasma glucose level $\geq 126 \mathrm{mg} / \mathrm{dL}(7.0 \mathrm{mmol} / \mathrm{L}), \mathrm{HbA} 1 \mathrm{c}$ level $\geq 6.5 \%$, or self-reported use of antihyperglycemic drugs.

the HDL-C level $(\mathrm{p}<0.0001)$ was observed in evacuees compared with non-evacuees (Table 3), and increases in the BMI $(\mathrm{p}<0.0001)$, diastolic blood pressure $(\mathrm{p}=0.006)$, LDL-C $(\mathrm{p}<$ $0.0001)$, AST $(p=0.016)$, ALT $(p<0.0001)$, and $\gamma$-GT levels $(\mathrm{p}<0.0001)$ were significantly greater in evacuees than nonevacuees.

\section{Risk factors for the incidence of hypo-HDL- cholesterolemia after disaster}

A multivariate logistic regression analysis was performed to identify independent associations among baseline risk factors and the incidence of hypo-HDL cholesterolemia after 
Table 5. Incidence of Hypo-HDL Cholesterolemia Following the Great East Japan Earthquake.

\begin{tabular}{|c|c|c|c|c|}
\hline \multirow{2}{*}{$\begin{array}{c}\mathrm{HDL}-\mathrm{C} \geq 40 \mathrm{mg} / \mathrm{dL} \text { before the } \\
\text { Earthquake }\end{array}$} & \multicolumn{4}{|c|}{ Incidence of hypo-HDL cholesterolemia $(<40 \mathrm{mg} / \mathrm{dL})$ after the Earthquake $(\mathrm{n})$} \\
\hline & Total & Non-evacuees & Evacuees & p value* \\
\hline Total $(\mathrm{n}=25,835)$ & $3.81 \%(985)$ & $3.23 \%(540)$ & $4.89 \%(445)$ & $<0.0001$ \\
\hline $\operatorname{Men}(\mathrm{n}=11,248)$ & $5.88 \%(661)$ & $4.93 \%(364)$ & $7.67 \%(297)$ & $<0.0001$ \\
\hline Women $(\mathrm{n}=14,587)$ & $2.22 \%(324)$ & $1.88 \%(176)$ & $2.83 \%(148)$ & 0.0002 \\
\hline
\end{tabular}

the disaster (Table 4). The evacuation was significantly associated with the incidence of hypo-HDL cholesterolemia [odds ratio (OR) 1.387, $\mathrm{p}<0.0001$ ] after adjusting for age, sex, BMI, smoking status, systolic blood pressure, diabetes, and ALT and $\gamma$-GT levels. The baseline BMI, an increase of BMI $\left(1 \mathrm{~kg} / \mathrm{m}^{2}\right)$, daily drinking, current smoking, ALT and $\gamma$ GT levels, and diabetes were also significantly associated with the incidence of hypo-HDL cholesterolemia (OR 1.106, $\mathrm{p}<0.0001$; OR 1.205, $\mathrm{p}<0.0001$; OR 0.709, $\mathrm{p}=0.00326$; OR 1.574, $\mathrm{p}<0.0001$; OR 1.384, $\mathrm{p}=0.0002$ and OR 0.791, $\mathrm{p}=$ 0.0004 ; and OR 1.284, $\mathrm{p}=0.0157$, respectively). In the gender-segregated multivariate logistic regression analysis, the evacuation was significantly associated with the incidence of hypo-HDL-cholesterolemia in both men (OR 1.413, $\mathrm{p}<0.0001$ ) and women (OR 1.351, p=0.0089) after adjusting for age, BMI, smoking status, systolic blood pressure, diabetes, and ALT and $\gamma$-GT levels. The baseline BMI, an increase of BMI $\left(1 \mathrm{~kg} / \mathrm{m}^{2}\right)$, and ALT were also significantly associated with the incidence of hypo-HDL cholesterolemia in both men (OR 1.129, p<0.0001; OR 1.197, p< 0.0001 ; and OR 1.238, $\mathrm{p}=0.0456$, respectively) and women (OR 1.074, p<0.0001; OR 1.222, p<0.0001; and OR 1.657, $\mathrm{p}=0.0011$, respectively). Additionally, daily drinking, current smoking, and $\gamma$-GT were also significantly associated with the incidence of hypo-HDL cholesterolemia in men (OR $0.714, \mathrm{p}=0.0406$; OR 1.618, $\mathrm{p}<0.0001$; and OR 0.766, $\mathrm{p}=$ 0.0005 , respectively).

\section{Incidence of hypo-HDL cholesterolemia after the di- saster}

The crude incidence of hypo-HDL cholesterolemia in the normal HDL-C level group (25,835 subjects) was $3.81 \%$ (985 subjects). Furthermore, the crude incidence of hypoHDL cholesterolemia was $5.88 \%$ (661 subjects) in men $(11,248$ subjects) and $2.22 \%$ (324 subjects) in women (14,587 subjects; Table 4). Specifically, in the normal HDLC level group (9,107 evacuees and 16,728 non-evacuees), the crude incidence of hypo-HDL cholesterolemia was significantly higher $(\mathrm{p}<0.0001$; Table 5$)$ in evacuees $(4.89 \%$; 445 subjects) than non-evacuees (3.23\%; 540 subjects). In addition, in men (3,871 evacuees and 7,377 non-evacuees), the crude incidence of hypo-HDL cholesterolemia was significantly higher $(\mathrm{p}<0.0001$; Table 4$)$ in evacuees $(7.67 \%$; 297 subjects) than non-evacuees $(4.93 \% ; 364$ subjects). In women (5,236 evacuees and 9,351 non-evacuees), the inci- dence of hypo-HDL cholesterolemia was also significantly higher $(\mathrm{p}=0.0002$; Table 5$)$ in evacuees $(2.83 \%$; 148 subjects) than non-evacuees (1.88\%; 176 subjects).

\section{Discussion}

The present study demonstrated that the prevalence of hypo-HDL cholesterolemia was significantly increased in evacuees compared to non-evacuees after the Great East Japan Earthquake and Fukushima Daiichi nuclear disaster. All metabolic factors including the BMI, blood pressure, glucose metabolism, lipid metabolism, and liver function were adversely affected in the normal HDL-C level group. In addition, the decrease in HDL-C was significantly greater in evacuees than non-evacuees in the normal HDL-C level group. Moreover, adverse effects on all metabolic factors, including the BMI, blood pressure, glucose metabolism, lipid metabolism, and liver function, were significantly more prevalent in male evacuees in the normal HDL-level group compared to non-evacuees. On the other hand, in the hypoHDL cholesterolemia group, the levels of HDL-C significantly increased after the disaster, however, the other metabolic factors, except glucose metabolism, were significantly and negatively affected. Furthermore, the present study also showed that the evacuation was significantly associated with the incidence of hypo-HDL cholesterolemia and the incidence of hypo-HDL cholesterolemia was significantly higher in evacuees than non-evacuees. Thus, this historic disaster was more likely to have a negative effect on metabolic laboratory test results in the evacuees group than the nonevacuees group. These findings suggest that chronic metabolic health problems such as obesity, type 2 diabetes, hypertension and dyslipidemia should be carefully monitored and treated after the disaster, especially in evacuees.

Substantial epidemiological studies have demonstrated that the HDL-C level is a strong, independent, and inverse risk factor of $\operatorname{CVD}(15,16)$ and $\operatorname{CVA}(5,6)$. Hypo-HDL cholesterolemia is strongly and inversely associated with the risk for coronary heart disease (17) and cerebrovascular disease (18). The high HDL-C level exerts its cardio-protective effect primarily through its role in reverse cholesterol transport and its anti-inflammatory, anti-thrombotic and antioxidative properties. As endogenous estrogens have a favorable effect on lipid metabolism, women often have higher HDLC levels than men (19). Additionally, alcohol consumption 
can increase the HDL-C levels. Various metabolic and environmental factors, including acute or chronic infection, a high intake of refined carbohydrates and cigarette smoking, can decrease the HDL-C levels. Importantly, a low HDL-C level is likely a secondary effect from components of metabolic syndrome: insulin resistance, obesity, high triglyceride dyslipidemia, and chronic low grade inflammation (20). A life as an evacuee in unfavorable conditions increases stress in terms of privacy, food availability, duty assignments, financial stability and health (21). Natural disasters have been shown to have a negative impact on metabolic factors (22). After the Great Hansin-Awaji Earthquake, glycemic control was aggravated in diabetic patients, and an association among chronic life-threatening stress and a worsening of metabolic control has been suggested (21). Similar effects have been reported with hypertension, with ambulatory blood pressure revealing that sympathetic activation is an important component of rising blood pressure after lifethreatening events and may trigger myocardial infarction (23).

Our data showed that the negative effects on metabolic factors were greater in evacuees than non-evacuees. These differences might be related to changes in lifestyle such as diet, physical activity, and life environment before and after the evacuation. It has been demonstrated that the change of lifestyle is strongly associated with the prevalence of hypoHDL cholesterolemia. However, our results were obtained from a follow-up period of only 1.6 years. Therefore, it is necessary to evaluate these effects by a further long-term follow-up.

In conclusion, this is the first report to reveal that the evacuation was associated with the risk of incidence of hypo-HDL cholesterolemia, leading to an increase in CVD. This information could be very important for periodic health checkups and lifestyle recommendations for evacuees in the future.

The findings and conclusions of this article are solely the responsibility of the authors and do not represent the official views of the Fukushima Prefectural Government.

The authors state that they have no Conflict of Interest (COI).

\section{The Fukushima Health Management Survey Group}

Hitoshi Ohto, Masafumi Abe, Shunichi Yamashita, Kenji Kamiya, Seiji Yasumura, Mitsuaki Hosoya, Akira Ohtsuru, Akira Sakai, Shinichi Suzuki, Hiroaki Yabe, Masasharu Maeda, Shirou Matsui, Keiya Fujimori, Tetsuo Ishikawa, Tetsuya Ohira, Tsuyoshi Watanabe, Hiroaki Satoh, Hitoshi Suzuki, Yukihiko Kawasaki, Atsushi Takahashi, Kotaro Ozato, Gen Kobayashi, Shigeatsu Hashimoto, Satoru Suzuki, Toshihiko Fukushima, Sanae Midorikawa, Hiromi Shimura, Hirofumi Mashiko, Aya Goto, Kenneth Eric Nollet, Shinichi Niwa, Hideto Takahashi, and Yoshisada Shibata.

\section{Financial Support}

This survey was supported by the National Health Fund for
Children and Adults Affected by the Nuclear Incident.

\section{References}

1. Yasumura S, Hosoya M, Yamashita S, et al. Study protocol for the Fukushima Health Management Survey. J Epidemiol 22: 375-383, 2012.

2. Van Lenten BJ, Hama SY, de Beer FC, et al. Anti-inflammatory HDL becomes pro-inflammatory during the acute phase response. Loss of protective effect of HDL against LDL oxidation in aortic wall cell cocultures. J Clin Invest 96: 2758-2767, 1995.

3. Navab M, Hama SY, Anantharamaiah GM, et al. Normal high density lipoprotein inhibits three steps in the formation of mildly oxidized low density lipoprotein: steps 2 and 3. J Lipid Res 41: 1495-1508, 2000.

4. Barter P, Gotto AM, LaRosa JC, et al. HDL cholesterol, very low levels of LDL cholesterol, and cardiovascular events. N Engl J Med 357: 1301-1310, 2007.

5. Wannamethee SG, Shaper AG, Ebrahim S. HDL-Cholesterol, total cholesterol, and the risk of stroke in middle-aged British men. Stroke 31: 1882-1888, 2000.

6. Soyama Y, Miura K, Morikawa Y, et al. High-density lipoprotein cholesterol and risk of stroke in Japanese men and women: the Oyabe Study. Stroke 34: 863-868, 2003.

7. Expert Panel on Detection Evaluation, and Treatment of High Blood Cholesterol in Adults. Executive Summary of the Third Report of the National Cholesterol Education Program (NCEP) Expert Panel on Detection, Evaluation, and Treatment of High Blood Cholesterol in Adults (Adult Treatment Panel III). JAMA 285: 2486-2497, 2001.

8. Sharrett AR, Ballantyne CM, Coady SA, et al. Coronary heart disease prediction from lipoprotein cholesterol levels, triglycerides, lipoprotein(a), apolipoproteins A-I and B, and HDL density subfractions: The Atherosclerosis Risk in Communities (ARIC) Study. Circulation 104: 1108-1113, 2001.

9. Lloyd-Jones D, Adams R, Carnethon M, et al. Heart disease and stroke statistics-2009 update: a report from the American Heart Association Statistics Committee and Stroke Statistics Subcommittee. Circulation 119: 480-486, 2009.

10. Chapman MJ, Assmann G, Fruchart JC, Shepherd J, Sirtori C. European Consensus Panel on H-C. Raising high-density lipoprotein cholesterol with reduction of cardiovascular risk: the role of nicotinic acid-a position paper developed by the European Consensus Panel on HDL-C. Curr Med Res Opin 20: 1253-1268, 2004.

11. Teramoto T, Sasaki J, Ishibashi $S$, et al. Executive summary of the Japan Atherosclerosis Society (JAS) guidelines for the diagnosis and prevention of atherosclerotic cardiovascular diseases in Japan: 2012 version. J Atheroscler Thromb 20: 517-523, 2013.

12. Ellison RC, Zhang Y, Qureshi MM, et al. Lifestyle determinants of high-density lipoprotein cholesterol: the National Heart, Lung, and Blood Institute Family Heart Study. Am Heart J 147: 529-535, 2004.

13. International guidelines for ethical review of epidemiological studies. Law Med Health Care 19: 247-258, 1991.

14. Committee of the Japan Diabetes Society on the Diagnostic Criteria of Diabetes Mellitus; Seino Y, Nanjo K, Tajima N, et al. Report of the committee on the classification and diagnostic criteria of diabetes mellitus. J Diabetes Investig 1: 212-228, 2010.

15. Gordon DJ, Probstfield JL, Garrison RJ, et al. High-density lipoprotein cholesterol and cardiovascular disease. Four prospective American studies. Circulation 79: 8-15, 1989.

16. Gordon T, Castelli WP, Hjortland MC, Kannel WB, Dawber TR. High density lipoprotein as a protective factor against coronary heart disease. The Framingham Study. Am J Med 62: 707-714, 1977.

17. Chirovsky DR, Fedirko V, Cui Y, Sazonov V, Barter P. Prospective 
studies on the relationship between high-density lipoprotein cholesterol and cardiovascular risk: a systematic review. Eur J Cardiovasc Prev Rehabil 16: 404-423, 2009.

18. Okamura $T$, Hayakawa $T$, Kadowaki $T$, et al. The inverse relationship between serum high-density lipoprotein cholesterol level and all-cause mortality in a 9.6-year follow-up study in the Japanese general population. Atherosclerosis 184: 143-150, 2006.

19. Barton M. Cholesterol and atherosclerosis: modulation by oestrogen. Curr Opin Lipidol 24: 214-220, 2013.

20. Kontush A, Chapman MJ. Functionally defective high-density lipoprotein: a new therapeutic target at the crossroads of dyslipide- mia, inflammation, and atherosclerosis. Pharmacol Rev 58: 342374, 2006.

21. Inui A, Kitaoka H, Majima M, et al. Effect of the Kobe earthquake on stress and glycemic control in patients with diabetes mellitus. Arch Intern Med 158: 274-278, 1998.

22. Fonseca VA, Smith H, Kuhadiya N, et al. Impact of a natural disaster on diabetes: exacerbation of disparities and long-term consequences. Diabetes Care 32: 1632-1638, 2009.

23. Suzuki S, Sakamoto S, Koide M, et al. Hanshin-Awaji earthquake as a trigger for acute myocardial infarction. Am Heart J 134: 974977, 1997.

(C) 2016 The Japanese Society of Internal Medicine http://www.naika.or.jp/imonline/index.html 